\title{
ESTIMATION OF THE USEFULNESS OF GLASSY CARBON ELECTRODE IN NON-AQUEOUS SOLVENTS POLARIZED TO HIGHER ANODIC POTENTIALS
}

\author{
LÁSZLÓ KISSa,b*, SÁNDOR KUNSÁGI-MÁTÉc
}

\begin{abstract}
In this paper suitability of glassy carbon electrode in numerous non-aqueous solvents used widely in electrochemistry (methanol, acetone, dichloromethane, nitrobenzene, nitromethane, 1-butanol, dimethyl sulfoxide, dimethyl formamide, tetrahydrofuran) was studied by changing the potential of preanodisation. The potential range studied in viewpoint of preanodisation effect was between 1 and $3 \mathrm{~V}$. At higher potentials, glassy carbon electrode deactivated in many solvents which was clearly demonstrated using the aqueous solution of a redox probe 1,4-dihydroxybenzene. Due to the low permittivity some solvents (ethyl acetate, chloroform) were investigated using a preanodisation at $3 \mathrm{~V}$ because of the significant ohmic drop and the surface state also changed. Results highlight the importance of this limitation caused by polarizing above $2 \mathrm{~V}$ and it must be considered when glassy carbon electrode is selected for the investigations carried out in these conditions.
\end{abstract}

Keywords: Glassy carbon; Preanodisation; Permittivity; Deactivation; Nonaqueous solvents

\section{INTRODUCTION}

During electrochemical studies of selected compounds in solution phase reproducibility of their voltammetric peak associated with each individual cycle helps to decide if it fouls the electrode or not. Usually when the voltammetric peaks decline continuously or rapidly the conclusion is that a fouling process takes place. In certain conditions both the electrode material

\footnotetext{
a University of Pécs, Department of General and Physical Chemistry, Ifjúság street 6, H-7624 Pécs, Hungary

b János Szentágothai Research Center, Ifjúság útja 20, Pécs H-7624, Hungary

c University of Pécs, Department of Organic and Pharmacological Chemistry, Honvéd street 1, Pécs H-7624, Hungary

*Corresponding author: kiss/@gamma.ttk.pte.hu
} 
and solvent may affect the reproducibility of measurements. Recently, we found that electrochemical behavior of $N, N^{\prime}$-diphenylguanidine can be very different depending on the solvent used [1]. In the electrochemical studies, many solvents are used which can provide an appropriate medium for the investigation of selected material or process. Many solvents prone to oxidation or reduction according to their chemical nature and estimation of usefulness of electrodes prepared from different materials is an essential information. Glassy carbon electrode has various properties which can affect its behavior in different electrolyte media during its use in electrochemistry. Usually, the surface of the glassy carbon electrode is covered by phenolic hydroxy groups and quinone moieties being capable for chemical modifications. The disturbance of certain components might cause undesired changes in the surface state. Contrarily, the modified glassy carbon surface in special conditions has advantageous properties which gained wide application in electroanalytical chemistry. The number of surface functional groups can be multiplied by several activation procedures which has been used for several decades to alter the surface properties favourable in improvement for analytical properties. There are recent developments also for activation of glassy carbon electrode [2-10].

In our earlier investigations glassy carbon electrode was studied in two solvents. Acetonitrile proved unreliable by polarizing this electrode to potentials higher than $2 \mathrm{~V}$ vs. Ag wire which was highlighted by investigating phenols [11]. In acetonitrile on platinum electrode at very high potentials a remarkable decrease of currents showed formation of products blocking the surface [12]. In the same conditions electrooxidation of some para-substituted phenols was studied on platinum electrode and reproducible voltammograms could be obtained. Therefore, platinum electrode is appropriate as standard if we want to exclude everything apart from the electropolymerization of the substrate indicated by the decline of the voltammetric peaks. In another work mesityl oxide was studied and it fouled strongly the surface of glassy carbon electrode since it could be electropolymerized [13]. Mesityl oxide showed also that the choice of electrode might strongly influence the structure and sometimes composition of polyphenol films. Electrooxidation of mesityl oxide also itself resulted in surface deactivation of glassy carbon electrode.

In this work, a large number of non-aqueous solvents were tested in conditions when polarization of glassy carbon electrode to higher anodic potentials is applied. The electrooxidation of several organic materials generates radicals which can be bound to the surface functional groups presenting on the surface of this type of electrode. Investigations focused on how these conditions affect the measurement of an appropriate redox probe considering the modified electrode's surface state. 


\section{RESULTS AND DISCUSSION}

In order to assess the effect of preanodisation in the different solvents on the reliability of glassy carbon electrode in giving reproducible current signals the anodic potential was changed between 1 and $3 \mathrm{~V}$ in $0.5 \mathrm{~V}$ increments with duration of $10 \mathrm{~s}$ in their solutions containing $0.1 \mathrm{M}$ tetrabutylammonium perchlorate (TBAP) supporting electrolyte. With the pretreated electrode in this way cyclic voltammograms were recorded between 0 and $1 \mathrm{~V}$ in the aqueous solution of $10 \mathrm{mM}$ 1,4-dihydroxybenzene. This compound gives reproducible current signals on the commonly used electrodes while it oxidizes to $p$-benzoquinone in a $2 \mathrm{e}^{-} / 2 \mathrm{H}^{+}$reaction. The ratios of peak current measured with the pretreated electrode and of peak current measured with the bare electrode were used for the evaluation. Data were collected for each solvents and at the different anodic pretreatment potentials. The electrode surface was renewed before all pretreatments according to the procedure described in the Experimental section. The normalized peak currents are displayed in Fig. 1. The figure is divided into two ( $a$ and $b$ ) parts to see more clearly the effect of the numerous solvents.
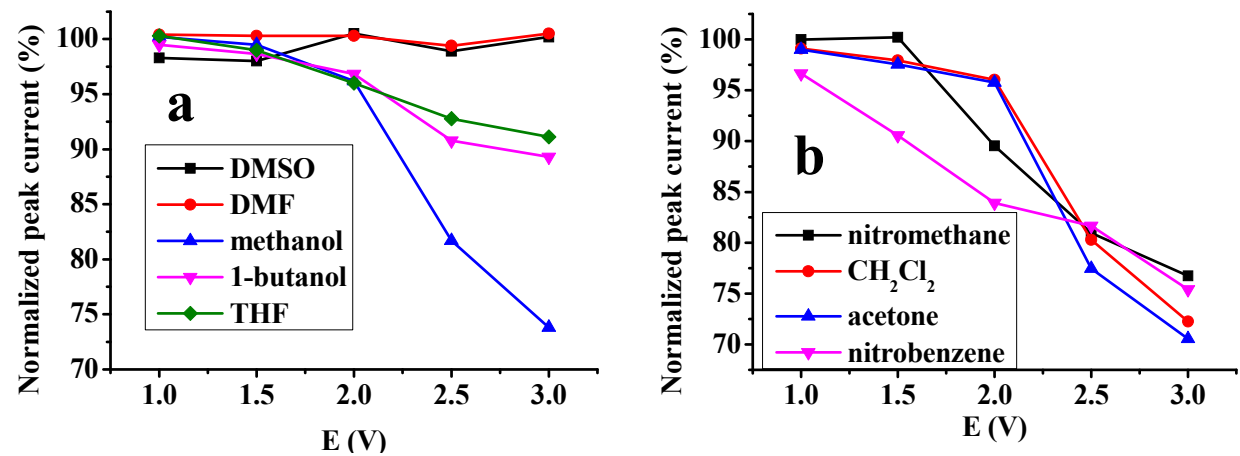

Figure 1. Normalized peak currents for aqueous solution of $10 \mathrm{mM}$ 1,4-dihydroxybenzene after $10 \mathrm{~s}$ preanodisation at potentials ranging from 1 to $3 \mathrm{~V}$ in the different solvents (supporting electrolyte $0.1 \mathrm{M} \mathrm{pH}=7$ phosphate buffer, scan rate $0.1 \mathrm{~V} / \mathrm{s}$ )

Dimethyl sulfoxide (DMSO) and dimethyl formamide (DMF) are susceptible to oxidation compared with the other solvents. Due to this fact their use is limited to mildly anodic potentials as they start to react below $2 \mathrm{~V}$ by using the commonly used electrodes [14]. Therefore, application of these solvents at high potentials has low interest. Despite of their usefulness in these conditions the effect of pretreatment potential was investigated up to 3 
V. Peak currents after the anodic pretreatments were identical to the value with subtle differences measured with the untreated electrode showing that glassy carbon surface does not change during the polarisation.

Alcohols can also serve as solvent especially the ones having larger molecular weight as their background currents are low according to our earlier findings obtained by investigation of phenol in solutions prepared with alcohol solvents [15]. Methanol produces significant currents above $1.5 \mathrm{~V}$ and therefore the electrochemical investigation of compounds seems very limited in this solvent. Moreover, glassy carbon surface deactivated remarkably by increasing the pretreatment potential. Increasing the molecular weight of alcohols, they start to oxidize at more and more positive potentials mainly due to the decrease of their dielectric constant. 1-butanol has broader potential window for experiments partly due to the lower dielectric constant. This property therefore resulted in lower current flows during the preanodisation steps compared to that measured in methanol. The normalized peak currents indicate electrode deactivation which however is smaller than in case of methanol. In presence of trace amount of water alcohols undergo predominantly oxidative dehydrogenation and the electrooxidation of produced aldehydes leads to the undesired surface modification which is faster in methanol.

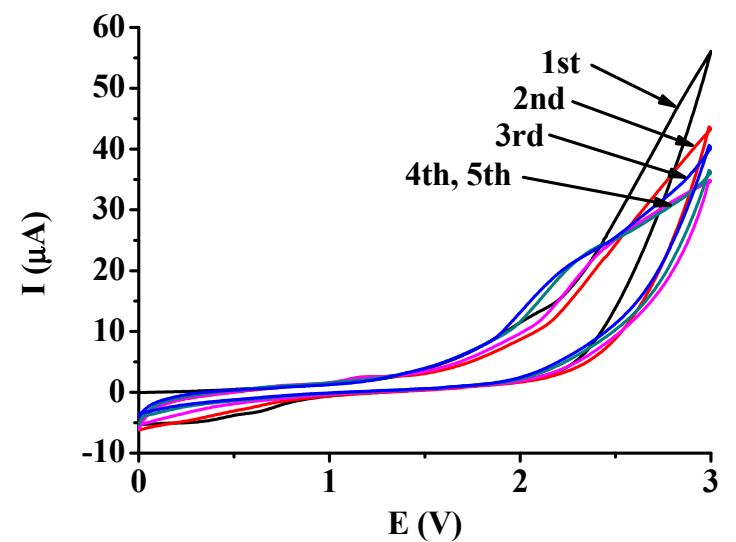

Figure 2. Voltammetric scans in nitrobenzene with glassy carbon electrode (supporting electrolyte $0.1 \mathrm{M}$ TBAP, scan rate $0.1 \mathrm{~V} / \mathrm{s}$ )

Nitrocompounds are not widespread in studying redox active compounds except for nitrobenzene which is used sometimes in electrochemistry. One of the possible reasons is its high toxicity. On the other hand, it has many advantages like high dielectric constant $(\varepsilon=35.6$ [16]), inertness towards oxidation at moderate anodic potentials, therefore the wide potential window 
in the anodic part offers wide applicability of this solvent. Additional limitations come from the lower solubility of a large number of analytes. The data show that glassy carbon electrode strongly passivates increasing the pretreatment potential in both nitrobenzene and in nitromethane. Fig. 2 displays additional curves of further cyclic voltammetric experiments conducted in nitrobenzene. It clearly shows also the deactivation process during the repeated voltammetric cycles. For making the fouling process deeper the potential of glassy carbon electrode was kept at $3 \mathrm{~V}$ for $60 \mathrm{~s}$ in nitrobenzene. According to the expectations peak current of 1,4-dihydroxybenzene in its aqueous solution was reduced to $45.78 \%$. For comparison the curves are displayed in Fig. 3. Interestingly, the anodic peak potential for oxidation of the redox probe was significantly smaller and cathodic potential shifted significantly to the positive direction suggesting some electrocatalytic behaviour of pretreated electrode but tortuosity effect is very significant seen from the drastically reduced peak currents. Therefore, the highly increased mass transport resistance leads to diminished analytical signals. The cathodic peak current was significantly higher indicating that the deposited layer has binding sites being favourable for adsorption of $p$-benzoquinone which is the oxidation product of the redox probe. Nitromethane contains a methyl group being able to oxidize at appropriately high potentials followed by the formation of a nitromethyl radical. They may interact with the surface hydroxy groups of glassy carbon so they can be bound covalently to the surface. The rate of surface coverage increases in the presence of these groups by increasing the pretreatment potential and then, a large number of redox active molecules can not reach the electrode surface.

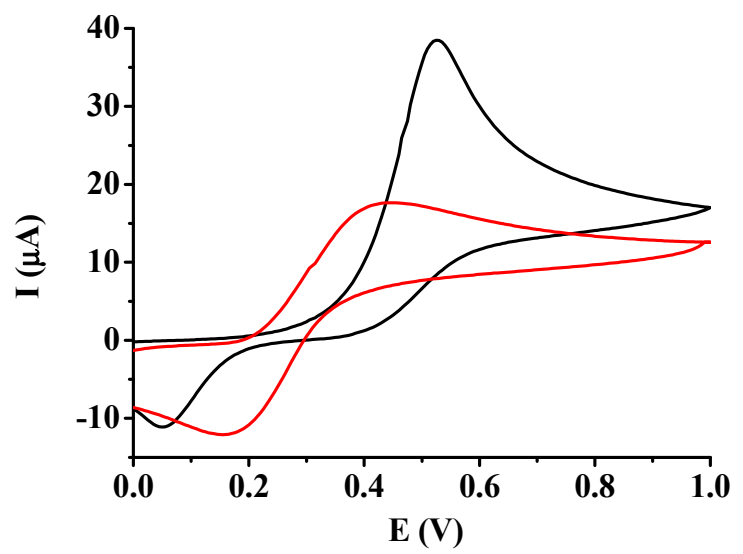

Figure 3. Voltammetric curves of $10 \mathrm{mM}$ 1,4-dihydroxybenzene with bare glassy carbon (black curve) and with the same preanodized electrode in nitrobenzene at $3 \mathrm{~V}$ (red curve) (supporting electrolyte $0.1 \mathrm{M} \mathrm{pH}=7$ phosphate buffer, scan rate $0.1 \mathrm{~V} / \mathrm{s}$ ) 
Acetone is not used widely as solvent in electrochemistry in contrast to its potential window which might be exploited as its oxidation starts around $2 \mathrm{~V}$ and the favourable permittivity allows relatively small ohmic potential drop. Its high volatility is very disadvantageous by the majority of applications in electrochemistry. Anodic polarization causes surface alteration as this solvent oxidizes on its methyl groups and the formed radicals can couple with each other forming oligomers and also can bound to the surface hydroxy groups but the latter process is responsible for the electrode destroying.

Tetrahydrofuran (THF) is also a very volatile liquid, moreover has a very low permittivity, therefore insufficient for the dissolving of salts. Moreover, the ohmic drop is significant so these properties make it unpopular as solvent for electrochemists. It is used widely as aprotic solvent and co-solvent in characterization of chemical processes mainly with spectroscopic methods. The results obtained with preanodisation in tetrahydrofuran show that use of glassy carbon would be limited also to the lower potentials.

Dichloromethane has a broad interest in the electrochemical investigations therefore effect of anodic potential during pretreatment in it might provide worthy information. The peak currents of redox probe indicate a remarkable decrease by increasing the pretreatment potential. The reason for that might be the oxidative cleavage of $\mathrm{C}-\mathrm{H}$ bonds and the subsequent radical formation can result chlorocarbons through their recombination and bound to the surface. The latter process enhances the surface resistivity therefore charge transfer reactions become inhibited.

There are low permittivity solvents used rarely as solvents in electrochemistry like ethyl acetate and chloroform only in combination with microelectrodes $[17,18]$. Therefore, it seems difficult to access their usefulness in their own liquid phase due to the very high degree of ionic association of supporting electrolyte. In case of these two solvents $60 \mathrm{~s}$ polarization was carried out at a constant potential $3 \mathrm{~V}$. Then the voltammograms recorded respectively in the aqueous solution of model compound were used for the estimation of surface deactivation. In ethyl acetate the peak current declined to $63.6 \%$ and in chloroform $64.2 \%$ and showed that the polarisation to $3 \mathrm{~V}$ can lead to visible changes.

\section{CONCLUSION}

Numerous non-aqueous solvents were examined according to their different effects on the electrochemical behavior measured by cyclic voltammetry. It was found that they deactivate the surface of glassy carbon electrode and the limitations are revealed in cases when this electrode is selected for the 
corresponding investigations. Nitrobenzene provided surprising findings as new layers can be prepared based on it giving alternatives for future investigations of modified electrodes. On the other hand, the investigation of other carbon based electrodes would be interesting regarding the effect of anodic pretreatment.

\section{EXPERIMENTAL}

For the electrochemical investigations analytical grade solvents were used. The supporting electrolyte was tetrabutylammonium perchlorate in the non-aqueous solvents and in aqueous solvents $\mathrm{pH}=7$ phosphate buffer was used. The used non-aqueous solvents had low moisture content. In all electrochemical experiments the working electrode was a glassy carbon disc sealed in polyetheretherketone (1 $\mathrm{mm}$ in diameter) and a Pt wire served as counter electrode. Saturated calomel electrode was used as reference in aqueous solutions. The equipment used for the electrochemical experiments was a potentiostat (Dropsens, Spain).

The surface of glassy carbon electrode was sanded off with 4000 grit emery paper and then polished with 1 and $0.05 \mu \mathrm{m}$ alumina slurry on a polishing cloth. Finally, it was thoroughly washed with twice deionized water. To minimize the introduction of water into the electrochemical cell the electrodes were thoroughly rinsed with dry acetone and allowed to dry completely.

\section{ACKNOWLEDGEMENTS}

Financial support of the GINOP 2.3.2-15-2016-00022 grant is highly appreciated.

\section{REFERENCES}

1. L. Kiss, F. Kovács, S. Kunsági-Máté, Periodica Polytechnica Chemical Engineering, https://doi.org/10.3311/Ppch.14959.

2. A.M. Abdel-Aziz, H.H. Hassan, I.H.A. Badr, Analytical Chemistry, 2020, 92, 7947.

3. A. Dekanski, J. Stevanovic, R. Stevanovic, B.Z. Nikolic, V.M. Jovanovic, Carbon, 2001, 39, 1195.

4. B. Farajmand, M.A. Kamyabi, F.Y. Sorkhani, H.S. Jam, H. Bahrami, Journal of Electroanalytical Chemistry, 2020, 861, DOI: 10.1016/j.jelechem.2020.113966. 
5. Y.W. Li, J. Zhou, J. Song, X.S. Liang, Z.P. Zhang, D. Men, D.B. Wang, X.E. Zhang, Biosensors \& Bioelectronics, 2019, 144, DOI: 10.1016/j.bios.2019.111534.

6. L.Y. Wang, Y. Wang, Q.F. Zhuang, Journal of Electroanalytical Chemistry, 2019, 851, DOI: 10.1016/j.jelechem.2019.113446.

7. T. Bystron, E. Sramkova, F. Dvorak, K. Bouzek, Electrochimica Acta, 2019, 299, 963.

8. S. Berto, L. Carena, F. Valmacco, C. Barolo, E. Conca, D. Vione, R. Buscaino, M. Fiorito, C. Bussi, O. Abollino, M. Malandrino, Electrochimica Acta, 2018, 284, 279.

9. X.B. Hu, W.H. Zheng, R.F. Zhang, Journal of Solid State Electrochemistry, 2016, 20, DOI: 10.1007/s10008-016-3302-8.

10. E. Chiavazza, S. Berto, A. Giacomino, M. Malandrino, C. Barolo, E. Prenesti, D. Vione, O. Abollino, Electrochimica Acta, 2016, 192, 139.

11. L. Kiss, S. Kunsági-Máté, Periodica Polytechnica Chemical Engineering, https://doi.org/10.3311/Ppch.14311.

12. J. Cassidy, S.B. Khoo, S. Pons, M. Fleischmann, Journal of Physical Chemistry, 1985, 89, 3933.

13. L. Kiss, F. Kovács, H. Li, A. Kiss, S. Kunsági-Máté, Chemical Physics Letters, 2020, 754 .

14. L. Kiss, D. Bősz, F. Kovács, H. Li, G. Nagy, S. Kunsági-Máté, Polymer Bulletin, 2019, 76, 5849.

15. L. Kiss, D. Bősz, F. Kovács, H. Li, S. Kunsági-Máté, Polymer Bulletin, 2019, 76, 215.

16. David R. Lide, Handbook of Chemistry and Physics, $76^{\text {th }}$ Edition 1995-1996, Chapter 6, p. 170.

17. M.A. Hernández-Olmos, L. Agüí, P. Yánez-Sedeno, J.M. Pingarrón, Electrochimica Acta, 2000, 46, 289.

18. L. Agüí, J.E. López-Guzmán, A. González-Cortés, P. Yánez-Sedeno, J.M. Pingarrón, Analytica Chimica Acta, 1999, 385, 241. 